\title{
Neutropenia Following Intravenous Immunoglobulin Administration in a Patient with Multifocal Motor Neuropathy with Conduction Block
}

Sung-Yeon Sohn* and Jae Guk Kim

Department of Neurology, Eulji University Hospital, Daejeon, Republic of Korea

*Corresponding author: Sung-Yeon Sohn, Department of Neurology, Eulji University Hospital 95, Dunsanseo-ro, Seo-gu, Daejeon, Republic of Korea; Tel: +82-42-611-3241; E-mail: sungyeonsohn@gmail.com

Received date: November 21, 2016; Accepted date: January 06, 2017; Published date: January 13, 2017

Copyright: (c) 2017 Sohn SY, et al. This is an open-access article distributed under the terms of the Creative Commons Attribution License, which permits unrestricted use, distribution, and reproduction in any medium, provided the original author and source are credited.

\begin{abstract}
Intravenous immunoglobulin (IVIG) is the treatment of choice in patients with multifocal motor neuropathy with conduction block (MMNCB). Although IVIG is considered generally safe, neutropenia following IVIG infusion has been recognized as a rare hematologic side effect. Herein we report a patient with MMNCB who developed severe neutropenia following IVIG administration.

A 41 year old woman presented with progressive weakness of her left lower extremity with frequent muscle cramps. Nerve conduction study revealed marked conduction blocks on right ulnar and right tibial nerves. Serum $\operatorname{lgM}$ anti-GM1 antibody was detected. Neutrophil count decreased from day 2 and recovered after 2 weeks following IVIG infusion. To our knowledge, neutropenia following IVIG infusion in MMNCB has never been discussed apart, although it can be an important clinical issue especially for patients with MMNCB.
\end{abstract}

Keywords: Multifocal motor neuropathy with conduction block; Neutropenia; Intravenous immunoglobulin

\section{Introduction}

Multifocal motor neuropathy with conduction block (MMNCB) is a rare chronic immune mediated polyneuropathy which prevalence is known to be less than 1 per 100000 [1]. Although rare, if recognized early and properly managed, $\mathrm{MMNCB}$ is a treatable condition. MMNCB should be distinguished from other chronic acquired demyelinating polyneuropathy, especially in that MMNCB is resistant to corticosteroid and plasmapheresis [2]. To date, intravenous immunoglobulin (IVIG) administration is the mainstay of MMNCB therapy owing to its excellent safety profile as well as its clinical efficacy $[3,4]$.

Intravenous immunoglobulin is generally considered safe [5], despite hematologic toxicities were recognized in various clinical applications, such as idiopathic thrombocytopenia purpura, systemic lupus erythematosus, pemphigus vulgaris, Guillain-Barre syndrome (GBS), chronic inflammatory demyelinating polyradiculoneuropathy (CIDP), polymyositis [6]. To our knowledge, leukopenia following IVIG administration in MMNCB has never been discussed apart, although it can be an important clinical issue especially for patients with MMNCB. We report herein a patient with MMNCB who developed leukopenia following IVIG infusion.

\section{Case Report}

A 41 year old woman visited our hospital complaining of insidious weakness in her left leg, which was worsening from 6 months ago. She had mild difficulty lifting up the left ankle, but it did not interfere with her daily activities. From 2 months before admission, she experienced frequent muscle cramps at night, and the frequency of nocturnal cramps increased over time. The patient denied any sensory symptoms.
Her previous medical history was unremarkable. On neurological examination, motor weakness was confined to her left leg, especially dorsiflexion of great toe and ankle showed Medical Research Council (MRC) grade 4+. Steppage gait was noticed while walking. Sensory examination to all modalities was normal. Deep tendon reflexes including left knee jerk were all within normal limits. Nerve conduction study revealed marked conduction blocks on right ulnar and right tibial nerves (Figure 1), with slowing of motor conduction velocities on left median, right ulnar, left peroneal nerves. Laboratory investigations for rheumatoid factor, antinuclear antibody, anti-Ro/La, anti-neutrophil cytoplasmic antibody, anti-myelin associated glycoprotein antibody, vitamin B12 level, protein electrophoresis and immunofixation electrophoresis showed negative results. Cerebrospinal fluid analysis showed normal protein level without pleocytosis.

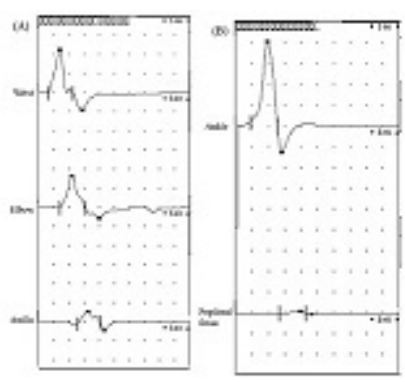

Figure 1: Nerve conduction study of the patient at presentation. Note the prominent motor conduction block of the right ulnar nerve between elbow and axilla (A) and of the right tibial nerve between ankle and popliteal fossa (B). 
Under the diagnosis of MMNCB, the administration of IVIG was initiated $(0.4 \mathrm{~g} / \mathrm{Kg} /$ day for 5 days). White blood cell count began to decrease from day 2 and showed nadir on day 4, reaching absolute neutrophil count of 370 cells/ $\mu \mathrm{L}$. The occurrence of leukopenia was thought to be due to neutropenia, since neutrophil count decreased most prominently, accounting for 22 percent of total leukocytes during nadir (Figure 2). White blood cell count recovered to normal range 2 weeks after completion of IVIG infusion. Serum IgM anti-GM1 antibody was detected at high titer $(>100 \%$ by enzyme-linked immunosorbent assay; Bühlmann Laboratories, Switzerland), confirming the diagnosis in our patient.

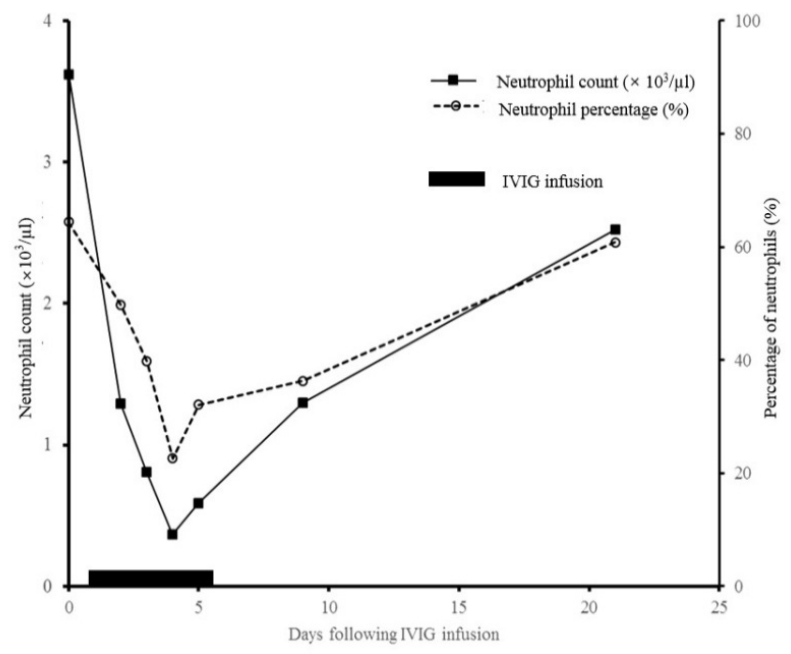

Figure 2: Changes in neutrophil count and neutrophil percentage before and after IVIG administration. IVIG was infused from day 1 to day 5 . Neutrophil count decreased to 370 cells/ $\mu \mathrm{L}$ on day 4 .

\section{Discussion}

Since the introduction of IVIG therapy in various immunological diseases, neutropenia was reported as a rare adverse event. Neutropenia following IVIG was initially recognized in patients with idiopathic thrombocytopenia purpura [7,8]. Ben-Chetrit et al. [9] reported a patient with active systemic lupus erythematosus who received two courses of IVIG infusion. Marked neutropenia developed repeatedly after each IVIG course, confirming the association between IVIG infusion and neutropenic event. In the present case, neutropenia developed on day 2 after initiation of infusion and reached nadir on day 4 . In line with previous reports $[8,10]$, leukocyte counts of our patient returned to baseline after 2 weeks without any complication.

The mechanism of neutropenia following IVIG remains to be elucidated. Tam et al. [11] reported a child with Guillain-Barre syndrome, in whom the authors revealed the presence of antineutrophil antibodies. Some authors suggested roles of atypical antineutrophil cytoplasmic antibodies (ANCA) [12,13]. IgG aggregates with immune-complex-like activity was suggested as the cause of transient neutropenia by some researchers $[9,14]$. Matsuda et al. [10] focused on two factors located in the surface of neutrophils: a complement receptor $\mathrm{CD} 11 \mathrm{~b}$ and an $\mathrm{Fc}$ gamma receptor CD16. Of the two surface antigens, only the level of expression of CD11b was found to be decreased after IVIG. The authors concluded that circulating neutrophils may adhere to vascular wall with the involvement of CD11b. More recently, von Gunten et al. $[15,16]$ demonstrated that neutropenia was mediated by sialic acid-binding immunoglobulin-like lectin 9 (siglec-9) expressed on neutrophils. IVIG preparations may contain natural autoantibodies to siglec- 9 and these autoantibodies can cause neutropenia in both caspase-dependent and independent manner.

The side effect of IVIG in MMNCB, including our case, should not be considered as similar to that seen in other neurological disorders. For instance, IVIG is widely used in GBS patients, but it is not necessary to continue IVIG administration despite side effect such as IVIG-induced neutropenia, since GBS usually does not recur and there are other treatments as plasmapheresis. In CIDP or polymyositis, alternative therapies such as corticosteroid or other immunosuppressive agents are still available. IVIG therapy is unique for MMNCB patients with no suitable alternative, since corticosteroids or plasmapheresis can lead to worsening of the disease and immunosuppressive agent like cyclophosphamide is limited owing to its cumulative toxicity [3]. Furthermore, long-term regular maintenance dosing of IVIG is required in most cases of MMNCB [2]. We found no articles discussing the long-term safety of repeated IVIG administration in patients with IVIG-induced neutropenia. If neutropenia occurs repeatedly, there is always possibility of exacerbating or causing sepsis, and some authors have expressed concerns about using IVIG in these situations [17].

To date, two approaches have been proposed to prevent IVIGinduced neutropenia. First, Matsuda et al. revealed that coadministration of corticosteroids prevented neutropenia following IVIG infusion [10], but that may not be appropriate for patients with MMNCB. Second, subcutaneous immunoglobulin (SCIG) may be an alternative $[18,19]$. Hadden et al. briefly described the occurrence of IVIG-induced neutropenia in three patients with MMNCB, which resolved after switching to SCIG [20]. We have not been able to administer SCIG in our case because it is not available in Korea. But it seems quite promising for MMNCB patients with previous history of IVIG-induced neutropenia if available.

Neutropenia following IVIG administration has been considered to be transient and self-limited. However, it may be an important clinical issue in MMNCB because long-term IVIG infusions are often required. Further studies on the mechanism of IVIG-induced neutropenia are required to manage patients with $\mathrm{MMNCB}$ properly.

\section{References}

1. Bromberg MB, Franssen H (2015) Practical rules for electrodiagnosis in suspected multifocal motor neuropathy. J Clin Neuromuscul Dis 16: 141-152.

2. Latov N (2014) Diagnosis and treatment of chronic acquired demyelinating polyneuropathies. Nat Rev Neurol 10: 435-446.

3. Lawson VH, Arnold WD (2014) Multifocal motor neuropathy: A review of pathogenesis, diagnosis, and treatment. Neuropsychiatr Dis Treat 10: 567-576.

4. Brannagan TH (2002) Intravenous gammaglobulin (IVIg) for treatment of CIDP and related immune-mediated neuropathies. Neurology 59: S33S40.

5. Katz U, Achiron A, Sherer Y, Shoenfeld Y (2007) Safety of intravenous immunoglobulin (IVIG) therapy. Autoimmun Rev 6: 257-259.

6. Baxley A, Akhtari M (2011) Hematologic toxicities associated with intravenous immunoglobulin therapy. Int Immunopharmacol 11: 1663-1667. 
Citation: Sohn SY, Kim JG (2017) Neutropenia Following Intravenous Immunoglobulin Administration in a Patient with Multifocal Motor Neuropathy with Conduction Block. J Neurol Neurophysiol 8: 409. doi:10.4172/2155-9562.1000409

Page 3 of 3

7. Veys PA, Macey MG, Owens CM, Newland AC (1988) Neutropenia following intravenous immunoglobulin. Br Med J (Clin Res Ed) 296: 1800 .

8. Berkovitch M, Dolinski G, Tauber T, Aladjem M, Kaplinsky C (1999) Neutropenia as a complication of intravenous immunoglobulin (IVIG) therapy in children with immune thrombocytopenic purpura: Common and non-alarming. Int J Immunopharmacol 21: 411-415.

9. Ben-Chetrit E, Putterman C (1992) Transient neutropenia induced by intravenous immune globulin. N Engl J Med 326: 270-271.

10. Matsuda M, Hosoda W, Sekijima Y, Hoshi K, Hashimoto T, et al. (2003) Neutropenia as a complication of high-dose intravenous immunoglobulin therapy in adult patients with neuroimmunologic disorders. Clin Neuropharmacol 26: 306-311.

11. Tam DA, Morton LD, Stroncek DF, Leshner RT (1996) Neutropenia in a patient receiving intravenous immune globulin. J Neuroimmunol 64: 175-178.

12. Jolles S, Deacock S, Turnbull W, Silvestrini R, Bunn C, et al. (1999) Atypical c-ANCA following high dose intravenous immunoglobulin. J Clin Pathol 52: 177-180.

13. Jarius S, Eichhorn P, Albert MH, Wagenpfeil S, Wick M, et al. (2007) Intravenous immunoglobulins contain naturally occurring antibodies that mimic antineutrophil cytoplasmic antibodies and activate neutrophils in a TNFalpha-dependent and Fc-receptor-independent way. Blood 109: 4376-4382.
14. O’Donnell J, Ahern M, Roberts T P, Bradley J (1988) Alteration in circulating neutrophil CR3 density associated with immunoglobulin infusion. Australian and New Zealand Journal of Medicine 18:95-100.

15. von Gunten S, Simon HU (2008) Natural anti-siglec autoantibodies mediate potential immunoregulatory mechanisms: Implications for the clinical use of intravenous immunoglobulins (IVIg). Autoimmun Rev 7: $453-456$.

16. von Gunten S, Schaub A, Vogel M, Stadler BM, Miescher S, et al. (2006) Immunologic and functional evidence for anti-siglec-9 autoantibodies in intravenous immunoglobulin preparations. Blood 108: 4255-4259.

17. Khan S, Sewell WA (2007) Risks of intravenous immunoglobulin in sepsis affect trial design. Ann Intern Med 147: 813-814.

18. Misbah SA, Baumann A, Fazio R, Dacci P, Schmidt DS, et al. (2011) A smooth transition protocol for patients with multifocal motor neuropathy going from intravenous to subcutaneous immunoglobulin therapy: An open-label proof-of-concept study. J Peripher Nerv Syst 16: 92-97.

19. Harbo T, Andersen H, Jakobsen J (2010) Long-term therapy with high doses of subcutaneous immunoglobulin in multifocal motor neuropathy. Neurology 75: 1377-1380.

20. Hadden RD, Marreno F (2015) Switch from intravenous to subcutaneous immunoglobulin in CIDP and MMN: Improved tolerability and patient satisfaction. Therapeutic Advances in Neurological Disorders 8: 14-19. 